Groupe d'Annecy

\author{
Laboratoire \\ d'Annecy-le-Vieux de \\ Physique des Particules
}

Groupe de Lyon

École Normale Supérieure de Lyon

\title{
EXERCISES IN EQUIVARIANT COHOMOLOGY
}

\author{
R. Stora \\ Laboratoire de Physique Théorique ENSLAPP \\ LAPP, Chemin de Bellevue, BP 110 \\ F-74941 Annecy-le-Vieux Cedex, France. \\ and \\ CERN, Theory Division, CH-1211 Genève 23, Switzerland
}

\begin{abstract}
Equivariant cohomology is a mathematical framework particularly well adapted to a kinematical understanding of topological gauge theories of the cohomological type. It also sheds some light on gauge fixing, a necessary field theory operation connected with the non compactness of the gauge group. The respective roles of fields and observables are emphasized throughout.
\end{abstract}

ENSLAPP-A-619/96

CERN-TH/96-279

October 1996

*Lectures given at the NATO ASI, "Quantum fields and quantum space-time", Cargèse, July 22-August 3, 1996.

†URA 14-36 du CNRS, associée à l'Ecole Normale Supérieure de Lyon et à l'Université de Savoie 


\section{INTRODUCTION}

Equivariant cohomology [1 $]^{-}[5]$ is at the core of the geometrical interpretation of the topological -more precisely, cohomological- field theories proposed by E. Witten in 1988 [6, 7]. The corresponding mathematical equipment also sheds some light on the gauge fixing procedure [8] familiar in the Lagrangian formulation of gauge theories.

The purpose of these notes is to update and, at times, provide alternatives to the second part of the 1994 Les Houches lectures by S. Cordes, G. Moore, S. Rangoolam [9].

The first part is devoted to definitions and basic facts related to equivariant cohomology. It relies on a systematic use of the Weil algebra, which not only streamlines many of the basic calculations, but is also of prime relevance in the geometrical framework for cohomological field theories. This is the subject of section 2 .

Section 3 gathers three exercises.

The first one consists of a quick derivation of the Mathaï Quillen formulae [3] which give special integral representations of the Thom class of a vector bundle [9]. It is accompanied by another similar formula which implements "Cartan's theorem 3" described in section 2 [1].

The second one describes a general procedure to construct universal observables pertaining to cohomological field theories.

The third one completes the construction of actions for cohomological models as occuring in integral representations of integrals over orbit spaces of cohomology classes of top dimension. These actions involve, among others, gauge fixing terms whose cohomological interpretaion is displayed as well. For the sake of definiteness the Yang Mills case is thoroughly discussed.

\section{EQUIVARIANT COHOMOLOGY. DEFINITIONS AND ELEMENTARY PROPERTIES [1]-[4]}

\section{Example 1}

Let $M$ be a smooth manifold, $\Omega^{*}(M)$ the differential forms on $M, d_{M}$ the exterior differential.

Let $\mathcal{G}$ be a connected Lie group, Lie $\mathcal{G}$ its Lie algebra, acting smoothly on $M . \lambda \in$ Lie $\mathcal{G}$ is represented by a vector field on $M, \underline{\lambda}$. For each $\omega \in \Omega^{*}(M)$, define

$$
\begin{aligned}
& i_{M}(\lambda) \omega=i(\underline{\lambda}) \omega \\
& \ell_{M}(\lambda) \omega=\ell(\underline{\lambda}) \omega
\end{aligned}
$$

where $i(\underline{\lambda})$ denotes the interior product by the vector field $\underline{\lambda}$, and $\ell(\underline{\lambda})$, the corresponding Lie derivative:

$$
\begin{aligned}
\ell_{M}(\lambda) & =\left[i_{M}(\lambda), d_{M}\right]_{+} \\
{\left[i_{M}(\lambda), i_{M}\left(\lambda^{\prime}\right)\right]_{+} } & =0 \\
{\left[\ell_{M}(\lambda), i_{M}\left(\lambda^{\prime}\right)\right]_{-} } & =i_{M}\left(\left[\lambda, \lambda^{\prime}\right]\right) \\
{\left[\ell_{M}(\lambda), \ell_{M}\left(\lambda^{\prime}\right)\right]_{-} } & =\ell_{M}\left(\left[\lambda, \lambda^{\prime}\right]\right)
\end{aligned} \quad \lambda, \lambda^{\prime} \in \operatorname{Lie} \mathcal{G}
$$

where $\left[\lambda, \lambda^{\prime}\right]$ is the bracket in Lie $\mathcal{G}$ (recall $\left.\left[\underline{\lambda}, \underline{\lambda}^{\prime}\right]_{\text {Lie }}=\left[\underline{\lambda, \lambda^{\prime}}\right]\right)$. 
Forms $\omega$ such that $i_{M}(\lambda) \omega=0 \forall \lambda \in$ Lie $\mathcal{G}$ are called horizontal.

Forms $\omega$ such that $\ell_{M}(\lambda) \omega=0 \forall \lambda \in$ Lie $\mathcal{G}$ are called invariant.

Forms which are both horizontal and invariant are called basic.

Basic cohomology is the de Rham cohomology restricted to basic forms which obviously span a subcomplex of the de Rham complex.

\section{Remark}

The term basic refers to the following situation: if $M$ is locally of the form $X \times \mathcal{G}$ with local coordinates $x \in X, y \in \mathcal{G}$, one can write locally

$$
\omega(x, y, d x, d y)=\Sigma \omega_{M N}(x, y) d x^{M} d y^{N}
$$

where $M$ and $N$ are multi indices.

Horizontality implies $N=0$.

Invariance implies $\omega_{M O}$ is independent of $y$.

Thus

$$
\omega_{\text {basic }}(x, y d x, d y)=\Sigma \omega_{M}(x) d x^{M}
$$

i.e. can be identified with a form on the base $X$.

\section{Example 2}

The Weil algebra of $\mathcal{G}: \mathcal{W}(\mathcal{G})$. One defines $\mathcal{W}(\mathcal{G})$ as a graded commutative differential algebra: as a vector space

$$
\mathcal{W}(\mathcal{G})=\wedge(\text { Lie } \mathcal{G})^{*} \otimes S\left((\text { Lie } \mathcal{G})^{*}\right)
$$

where $\wedge(\text { Lie } \mathcal{G})^{*}$ is the Grassmann algebra of $(\text { Lie } \mathcal{G})^{*}$ and $S\left((\text { Lie } \mathcal{G})^{*}\right)$ is the symmetric tensor algebra of $(\text { Lie } \mathcal{G})^{*} \cdot \mathcal{W}(\mathcal{G})$ is generated by Lie $\mathcal{G}$ valued generators $\omega\left(\right.$ for $\left.\wedge(\text { Lie } \mathcal{G})^{*}\right)$, assigned grading $1, \Omega$ (for $S\left((\text { Lie } \mathcal{G})^{*}\right)$ ), assigned grading 2 . One defines the differential $d_{W}$ by

$$
\begin{aligned}
& d_{W} \omega=\Omega-\frac{1}{2}[\omega, \omega] \\
& d_{W} \Omega=-[\omega, \Omega] .
\end{aligned}
$$

\section{Remark}

Eqs (2.6) are an algebraic abstraction of the relationship between the "curvature" $\Omega$ of a connection $\omega$ on a principal $\mathcal{G}$ bundle, and the latter: the first one defines the curvature; the second one is the Bianchi identity " $d_{W}^{2}=0$ ", via the Jacobi identity $[\omega,[\omega, \omega]]=0$.

An action of Lie $\mathcal{G}$ on $\mathcal{W}(\mathcal{G})$ is defined by

$$
\begin{aligned}
& i_{W}(\lambda) \omega=\lambda \quad i_{W}(\lambda) \Omega=0 \\
& \ell_{W}(\lambda) \omega=[\lambda, \omega] \quad \ell_{W}(\lambda) \Omega=[\lambda, \Omega] .
\end{aligned}
$$

$i_{W}(\lambda), \ell_{W}(\lambda), d_{W}$ fulfill the analogs of Eqs(2.2) with the subscript $W$ replacing the subscript $M$. 
The cohomology of $\mathcal{W}(\mathcal{G})$ is easily seen to reduce to the scalars. Introduce for instance the homotopy [10]

$$
k=\int_{0}^{1} k_{t} \varphi_{t}
$$

where

$$
\varphi_{t}=\omega_{t} \frac{\partial}{\partial \omega}+\Omega_{t} \frac{\partial}{\partial \Omega}
$$

with, e.g.

$$
\omega_{t}=t \omega \quad \Omega_{t}=d_{W} \omega_{t}+\frac{1}{2}\left[\omega_{t}, \omega_{t}\right]
$$

and

$$
k_{t} \Omega_{t}=d_{t} \omega_{t} \quad k_{t} \omega_{t}=0 .
$$

One easily proves

$$
k_{t} d_{W}+d_{W} k_{t}=d_{t}=d t \frac{\partial}{\partial t} .
$$

The basic cohomology, on the other hand consists of all the $\mathcal{G}$ invariant polynomials in $\Omega$.

\section{Example 3}

$\left(E, d_{E}\right)$ is a graded commutative differential algebra with an action $i_{E}(\lambda), \ell_{E}(\lambda)$ of Lie $\mathcal{G}$ fulfilling relations (2.2) (with the subscript $M$ replaced by $E$ ). Examples 1 and 2 are particular cases of this.

Equivariant cohomology [1, 国.

Def:: The equivariant cohomology of $\left(E, d_{E}\right),\left(i_{E}(\lambda), \ell_{E}(\lambda)\right)$ is defined as the basic cohomology of $\left(\mathcal{W}(\mathcal{G}) \otimes E, D_{W}=d_{W}+d_{E}\right), I_{W}\left((\lambda)=i_{W}(\lambda)+i_{E}(\lambda), L_{W}(\lambda)=\ell_{W}(\lambda)+\ell_{E}(\lambda)\right)$.

This is the so called Weil model for equivariant cohomology.

As we shall see later, the intermediate model to be presently defined is of substantial interest in practical computations. The following construction is due to J. Kalkman [4]. It owes its simplicity to the systematic use of the Weil algebra and not of concrete connections.

Under the automorphism

$$
x \rightarrow e^{i_{E}(\omega)} x
$$

the differential $D_{W}$ goes into

$$
D_{i n t}=d_{W}+d_{E}+\ell_{E}(\omega)-i_{E}(\Omega)
$$

and the operation is transformed into

$$
\begin{aligned}
I_{\text {int }}(\lambda) & =i_{W}(\lambda) \\
L_{\text {int }}(\lambda) & =\ell_{W}(\lambda)+\ell_{E}(\lambda) .
\end{aligned}
$$

The most efficient way to do these calculations is to look at the family $D_{t}, I_{t}(\lambda), L_{t}(\lambda)$ conjugate to the initial data through the automorphism $x \rightarrow e^{t i_{E}(\omega)} x$. See also ref [5]. As we shall see later, the efficiency of this model is due to the simplicity of $I_{\text {int }}(\lambda)=i_{W}(\lambda)$. 


\section{Remark}

If $\omega, \Omega$ were replaced by a connection $\tilde{\omega}_{E}$ on $E$ (whose definition will be given later) and its curvature $\tilde{\Omega}_{E}$, the whole construction would be spoiled!

It is fairly easy to see that the basic cohomology, in this scheme, is realized on the $\mathcal{G}$ invariant elements of $S\left((\mathrm{Lie} \mathcal{G})^{*}\right) \otimes E$ endowned with the Cartan differential $D_{C}=d_{E}-$ $i_{E}(\Omega)$. ( $D_{C}^{2}=\ell_{E}(\Omega)$ vanishes on $\mathcal{G}$ invariant elements). The Cartan model is the most popular, although not always the most convenient.

\section{Remarks}

As mentioned earlier, basic cohomology is related to the "cohomology of the base" when the latter exists e.g., in the case of the action of $\mathcal{G}$ on a manifold $M$, when $M / \mathcal{G}$ exists as a manifold, i.e. when $M$ is a principal $\mathcal{G}$ bundle. This situation is characterized by the existence of $\mathcal{G}$ connections on $M$, i.e. Lie $\mathcal{G}$ valued one forms $\tilde{\omega}$ such that $i_{M}(\lambda) \tilde{\omega}=\lambda \quad \ell_{M}(\lambda) \tilde{\omega}=[\lambda, \tilde{\omega}]$. This generalizes to arbitrary commutative differential algebras $\left(E, d_{E}\right)$ for which a connection $\tilde{\omega}$ is defined as a Lie $\mathcal{G}$ valued element of grading one such that $i_{E}(\lambda) \tilde{\omega}=\lambda \ell_{E}(\lambda) \tilde{\omega}=[\lambda, \tilde{\omega}]$. In fact, in this case, equivariant cohomology is isomorphic with basic cohomology. This is Cartan's "theorem 3" [1, 5], which implies in particular that this holds independently of the choice of a connection $\tilde{\omega}$.

The proof is easy. First a basic cohomology class is obviously an equivariant cohomology class. Conversely, given an equivariant cohomology class with representative $P(\omega, \Omega, x), x \in E$ we have, using the homotopy [10,11] of the Weil algebra

$$
P(\omega, \Omega, x)-P(\tilde{\omega}, \tilde{\Omega}, x)=\int_{0}^{1} k_{t}\left(D_{W} P\right)\left(\omega_{t}, \Omega_{t}, x\right)+D_{W}\left(k_{t} P\right)\left(\omega_{t} \Omega_{t}, x\right)
$$

where now

$$
\begin{gathered}
D_{W}=d_{W}+d_{E} \\
\omega_{t}=t \omega+(1-t) \tilde{\omega} .
\end{gathered}
$$

The conclusion follows from

$$
\begin{gathered}
I_{W}(\lambda) \omega_{t}=\left[i_{W}(\lambda)+i_{E}(\lambda)\right] \omega_{t}=\lambda . \\
L_{W}(\lambda) \omega_{t}=\left[\ell_{W}(\lambda)+\ell_{E}(\lambda)\right] \omega_{t}=\left[\lambda, \omega_{t}\right]
\end{gathered}
$$

Before concluding this section, we should mention that we have described equivariant cohomology at the Lie algebra level as it was introduced by H. Cartan [1]. It only coïncides with that defined in terms of the classifying space of $\mathcal{G}$ when $\mathcal{G}$ is compact [1]. Although global phenomena have to be kept in mind we shall stick here to the infinitesimal description in view of the forthcoming applications to cohomological field theory models. 


\section{THREE EXERCISES}

The main leitmotiv of this section is the integral representation of the Dirac current located at the origin of a vector space $V$ :

$$
\Omega_{0} \equiv \delta(v) \wedge d v=N_{0} \int \mathcal{D} b \mathcal{D} \bar{\omega} e^{-i<b, v>+<\bar{\omega}, d v>}
$$

where $v$ denotes a set of coordinates in $V, \wedge d v$ denotes the corresponding volume form, $b$ denotes a set of dual coordinates in the dual $V^{*}, \bar{\omega}$ denotes the corresponding set of generators of $\wedge V^{*}, \mathcal{D} b=\wedge d b, \mathcal{D} \bar{\omega}$ is the volume element for Berezin integration, $<,>$ denotes the duality pairing, $N_{0}$ is a normalization constant only depending on $|V|$, the dimension of $V$.

This Dirac current is invariant under linear changes of coordinates, being a product of a bosonic and a fermionic $\delta$ function.

Another suggestive representation is:

$$
\Omega_{0} \equiv \delta(v) \wedge d v=N_{0} \int \mathcal{D} b \mathcal{D} \bar{\omega} e^{s(<\bar{\omega}, v>)}
$$

where $s$ is defined as

$$
s v=d v \quad s d v=0
$$

and is extended to the integration variables by

$$
s \bar{\omega}=i b \quad s b=0 .
$$

Other representatives of the top cohomology of $V$ with compact supports are obtained by perturbing the function under the $s$ operation

$$
\Omega=N_{0} \int \mathcal{D} \bar{\omega} \mathcal{D} b \quad e^{-s(<\bar{\omega}, v>-i<\bar{\omega}, \varphi(b)>)}
$$

for $\varphi$ 's such that $\langle b, \varphi(b)\rangle$ is positive at infinity and $\varphi(0)$ is bounded.

Exercise 1: The Thom class of a vector bundle [3, 4, 9] and Cartan's theorem 3 [1].

Let $E(M, V)$ be a vector bundle with fiber $V$ associated to a principal $\mathcal{G}$ bundle $P(M, \mathcal{G})$ :

$$
E(M, V)=P(M, \mathcal{G}) \times_{\mathcal{G}} V
$$

for some representation $R_{V}$ of $\mathcal{G}$ in $V$ with differential $t_{V}$ representing Lie $\mathcal{G}$.

The Dirac current on $V$ defines a distributional form on $E$ representing the "Poincaré dual" [9] of the section $V=0$ of $E$, diffeomorphic to $M$. Smooth representatives of this cohomology class with fast decrease along the fibers have been constructed by V. Mathaï and D. Quillen [3].

This can be compactly described as follows: construct in the intermediate model the equivariant class which extends a gaussian thickening of the Dirac current, namely $e^{-\frac{(v, v)}{2}} \wedge d v$, where $(v, v)$ is a $\mathcal{G}$ invariant form on $V$. This can be written as

$$
\Theta_{\text {int }}=N_{0} \int \mathcal{D} b \mathcal{D} \bar{\omega} e^{s^{t o p}\left(<\bar{\omega}, v>-i(\bar{\omega}, b)^{*}\right)}
$$


where

$$
\begin{aligned}
s^{\text {top }} V & =\psi_{V}+t_{V}(\omega) v \\
s^{\text {top }} \psi_{V} & =t_{V}(\omega) \psi-t_{V}(\Omega) V \quad \psi_{v}=d v \\
s^{\text {top }} \omega & =\Omega-\frac{1}{2}[\omega, \omega] \\
s^{\text {top }} \Omega & =-[\omega, \Omega] \\
s^{\text {top }} \bar{\omega} & =i b-\bar{\omega} t_{V}(\omega) \\
s^{\text {top }} i b & =i b t_{V}(\omega)-\bar{\omega} t_{V}(\Omega)
\end{aligned}
$$

and $(,)^{*}$ is the invariant form on $V^{*}$ which yields $($,$) on V$. In the Weil model, one only needs to replace $\psi_{V}=d v$ by $\psi_{V}=d v-t_{V}(\omega) v$.

The extension of $s^{t o p}$ to the integration variables not only allows a compact writing but also provides easy proofs that $\Theta$ is equivariantly closed. $\Theta$ is called the universal Thom class of $E$. Replacing $\omega$ by $\tilde{\omega}$, a connection on $P(M, \mathcal{G})$ and $\Omega$ by $\tilde{\Omega}$, the curvature of $\tilde{\omega}$ provides a globally defined form on $E$, the Thom class of $E$. By construction, smooth deformations of the function under the $s^{\text {top }}$ sign leave one in the same cohomology class. So does a variation of $\tilde{\omega}$. Replacing $v$ by $v(x), x \in M$, a smooth section of $E$ produces a cohomology class of $M$ located at the zeroes of that section, the so-called Euler class of $E$, independent of the choice of the section $v=v(x)$.

Another construction of the same type provides the identity which implements Cartan's theorem 3:

$$
\int \mathcal{D} \omega \mathcal{D} \Omega \delta(\omega-\tilde{\omega}) \delta(\Omega-\tilde{\Omega})=1 .
$$

Introducing integral representations for both the fermionic and the bosonic $\delta$ functions, we get

$$
\int \mathcal{D} \omega \mathcal{D} \Omega \mathcal{D} \bar{\omega} \mathcal{D} \bar{\Omega} e^{\bar{\omega}(\omega-\tilde{\omega})+i \bar{\Omega}(\Omega-\bar{\Omega})}=1 .
$$

Extending $s^{\text {top }}$ as usual to $P(M, \mathcal{G})$ and, to the integration variables, by

$$
\begin{aligned}
s i \bar{\Omega} & =\bar{\omega}+[\omega, i \bar{\Omega}] \\
s \bar{\omega} & =-[\Omega, i \bar{\Omega}]+[\omega, \bar{\omega}] .
\end{aligned}
$$

This can be written

$$
\int \mathcal{D} \omega \mathcal{D} \Omega \mathcal{D} \bar{\omega} \mathcal{D} \bar{\Omega} e^{s^{t o p} i \bar{\Omega}(\omega-\bar{\omega})}=1
$$

One may define $s^{\text {top }}$ on $P$ by

$$
\begin{aligned}
s^{t o p} p & =\psi_{p}+\mathcal{O}_{p}(\omega, p) \\
s^{t o p} \psi_{p} & =-\mathcal{O}_{p}(\Omega, p)-\frac{\partial \theta p}{\partial p}(\omega, p) \cdot \psi_{p}
\end{aligned}
$$

where $p$ denotes a set of coordinates on $P$. This implies

$$
\begin{aligned}
s^{t o p} \tilde{\omega} & =\tilde{\Omega}-\frac{1}{2}[\tilde{\omega}, \tilde{\omega}] \\
s^{t o p} \tilde{\Omega} & =-[\tilde{\omega}, \tilde{\Omega}] .
\end{aligned}
$$


If one defines $\tilde{\omega}$ by

$$
H(p) \psi_{p}=0
$$

where

$$
\psi_{p}=d p+\mathcal{O}(\tilde{\omega}, p)
$$

and $H(p) \psi_{p}$ has values in Lie $\mathcal{G}$, one also has

$$
\int \mathcal{D} \omega \mathcal{D} \Omega \mathcal{D} \bar{\omega} \mathcal{D} \bar{\Omega} \quad e^{s^{t o p}\left(i \bar{\Omega} H(p) \psi_{p}\right)}=1 .
$$

Exercise 2: Universal observables for cohomological models as equivariant characteristic classes [5, 12, 13].

We return to the situation where the manifold $M$ is smoothly acted on by the connected Lie group $\mathcal{G}$. Assume the action of $\mathcal{G}$ can be lifted to a $K$ principal bundle $P(M, K)$ over $M$ on which there is a $\mathcal{G}$ invariant $K$ connection $\Gamma$.

Define the equivariant curvature in the intermediate model

$$
R_{\text {int }}^{e q}(\Gamma)=D_{\text {int }} \Gamma+\frac{1}{2}[\Gamma, \Gamma]=R(\Gamma)-i_{P}(\Omega) \Gamma
$$

and the corresponding $K$-characteristic classes, $\mathcal{P}_{K}\left(R_{i n t}^{e q}(\Gamma)\right)$ where the $\mathcal{P}_{K}$ 's are $K$ invariant symmetric polynomials on Lie $K$. It is easy to prove that these define equivariant classes of $M$ independent of the choice of $\Gamma$. These can be written down in the Weil model upon operating with $e^{i_{P}(\omega)}$.

It turns out that these classes exhaust the examples of observables known for cohomological gauge models [6].

The case of the topological Yang Mills theory in four dimensions is well known. Let $\mathcal{A}$ be the space of connections on $P(M, G)$. On $P(M, G) \times \mathcal{A}$, one takes the invariant $\mathcal{G}$ connection $\underline{a}$ (considered as a one form on $P$ and a zero form -coordinate function- on $\mathcal{A}$ ). The equivariant curvature is, in the intermediate model

$$
\begin{aligned}
R_{\text {int }}^{e q}(\underline{a}) & =F(a)+\delta a+\Omega \\
& =F(a)+\psi_{\text {int }}+\Omega .
\end{aligned}
$$

In the Weil model $\underline{a}$ is transformed into $\underline{a}+\omega$

$$
R_{w}^{e q}(\underline{a}+\omega)=F(a)+\psi_{w}+\Omega .
$$

Taking a $G$ invariant symmetric polynomial on Lie $G P_{G}$ and expanding $P_{G}\left(R_{w}^{e q}\right)$ into monomials $P_{4-\mathrm{g}}^{\mathrm{g}}$ of bidegree $4 \mathrm{~g}$ on $M$, g on $\mathcal{A}$, yields observables upon integration over a cycle $\gamma_{4-\mathrm{g}}$ of dimension 4-g in $M$ :

$$
\mathcal{O}_{4-\mathrm{g}}^{\mathrm{g}}\left(\gamma_{4-\mathrm{g}}\right)=\int_{\gamma_{4-\mathrm{g}}} P_{4-\mathrm{g}}^{\mathrm{g}}
$$

whose cohomology class only depends on the homology class of $\gamma_{4-g}$. 
Exercise 3: Cohomological models and integral representations of orbit space integrals of top cohomology classes: Yang Mills theories [6, 14, 16, 17, 21].

The problem of integrating basic cohomology classes over orbit space is inherent to the present field theory formulations of gauge theories. Dynamical gauge theories are defined via a $\mathcal{G}$ invariant top form on a principal $\mathcal{G}$ bundle. For instance, Yang Mills theories are defined on $\mathcal{A}=P(\mathcal{A} / \mathcal{G}, \mathcal{G})$ the space of connections $\underline{a}$ on a principal bundle $P(M, G)$ where $G$ is a compact Lie group, and $\mathcal{G}$ its gauge group, which is non compact. The Yang Mills form

$$
\Omega_{Y M}=e^{-S_{i n v}(a)} \mathcal{D} a
$$

defines the dynamics, but is not integrable in the fiber direction. One wishes to integrate gauge invariant observables $\mathcal{O}_{i n v}(a) \Omega_{Y M}$. In most textbooks, the Faddeev Popov [8] gauge fixing procedure is presented by factoring out the volume of the gauge group. In J. Zinn-Justin's book [18] on the other hand this is achieved by "integrating over the fiber" a route we shall now follow. Given a $\mathcal{G}$ invariant volume form $\mu$ on $\mathcal{G}$ and its dual $\tilde{\mu}_{\mathcal{G}}$ on Lie $\mathcal{G}$ normalized so that

$$
<\mu_{\mathcal{G}}, \tilde{\mu}_{\mathcal{G}}>=1
$$

one can construct the Ruelle Sullivan [19] closed basic form

$$
\Omega_{R S}=i\left(\underline{\tilde{\mu}}_{\mathcal{G}}\right) \Omega_{Y M}
$$

where $\underline{\tilde{\mu}}_{\mathcal{G}}$ is obtained by substituting for each element $X_{\alpha}$ of Lie $\mathcal{G}$ the corresponding fundamental vector field $\underline{X}_{\alpha}$ on $\mathcal{A}$. Closedness is a consequence of both the closedness of $\Omega_{Y M}$ and of its invariance. Horizontality is obvious as well as invariance.

Both $\mathcal{O}_{\text {inv }}(a)$ and $\Omega_{R S}$ define objects on $\mathcal{A} / \mathcal{G}$, respectively a function and a top form denoted $\tilde{\mathcal{O}}(a), \tilde{\Omega}_{R S}$, and one wishes to integrate $\tilde{\mathcal{O}}(a) \tilde{\Omega}_{R S}$ over $\mathcal{A} / \mathcal{G}$. Choosing a locally finite covering $U_{i}$ of $\mathcal{A} / \mathcal{G}$ and a partition of unity $\theta_{i}(\dot{a})$ subordinate to it as well as local sections $\sigma_{i}$ defined by local equations

$$
g_{i}(a)=0
$$

above each $U_{i}$, we may write

$$
\begin{aligned}
<\mathcal{O}> & =\int_{\mathcal{A} / \mathcal{G}} \tilde{\mathcal{O}}(a) \tilde{\Omega}_{R S}=\sum_{i} \int_{\mathcal{A} / \mathcal{G}} \theta_{i}(\dot{a}) \mathcal{O}(a) \Omega_{R S} \int_{\text {fiber }} \delta\left(g_{i}\right) \wedge \delta g_{i} \\
& =\int_{\mathcal{A}} \sum_{i} \theta_{i}(\dot{a}) \delta\left(g_{i}\right) \wedge \delta g_{i} \mathcal{O}(a) \Omega_{R S}
\end{aligned}
$$

Gauge independence is due to the closedness and basicity of $\theta_{i}(\dot{a}) \mathcal{O}(a) \Omega_{R S}$ : at the infinitesimal level $\delta\left(g_{i}\right) \wedge \delta g_{i}$ varies by $\ell(\hat{\omega})\left[\delta\left(g_{i}\right) \wedge \delta g_{i}\right]$ where $\hat{\omega}$ is the vertical vector field defined by

$$
\ell_{\hat{\omega}} g_{i}=\Delta g_{i}
$$

where $\Delta$ denotes the infinitesimal change of section. The differential form

$$
\gamma=\sum_{i} \theta_{i}(\dot{a}) \delta\left(g_{i}\right) \wedge \delta g_{i}
$$


will be called the gauge fixing form. It has the following property: its projection $\hat{\gamma}$ on any fiber [20], i.e., the representative of its class modulo the ideal generated by horizontal forms of strictly positive degree is a top class of $\mathcal{G}$ whose integral is one:

$$
\int_{\text {Fiber }} \hat{\gamma}=1
$$

It therefore projects on the top cohomology class of $\mathcal{G}$ with compact supports (or fast decrease), normalized to 1 . Since $\Omega_{R S}$ is a top basic form, it is clear that only the fiber projection of $\gamma$ matters. $\gamma$ may be considered as the Poincaré dual of a section which does not exist.

So, we may as well represent our integral as

$$
<\mathcal{O}>=\int_{\mathcal{A} / \mathcal{G}} \tilde{\mathcal{O}}(a) \tilde{\Omega}_{R S}=\int_{\mathcal{A}} \gamma \mathcal{O}(a) \Omega_{R S}=\int_{\mathcal{A}} \hat{\gamma} \mathcal{O}(a) \Omega_{R S}
$$

where $\hat{\gamma}$ can be defined by choosing a connection $\tilde{\omega}$ :

$$
\hat{\gamma}=\gamma(a) \mu_{\mathcal{G}}(\tilde{\omega})
$$

so that

$$
<\mathcal{O}>=\int_{\mathcal{A} / \mathcal{G}} \tilde{\mathcal{O}}(a) \tilde{\Omega}_{R S}=\int_{\mathcal{A}} \gamma(a) \mathcal{O}(a) \Omega_{Y M} .
$$

This can be algebraized as

$$
<\mathcal{O}>=\int \tilde{\mu}_{\mathcal{G}}(\mathcal{D} \omega) \hat{\gamma}(a, \omega) \mathcal{O}(a) \Omega_{Y M} .
$$

where a Berezin integral over Lie $\mathcal{G}$ is included, and

$$
\hat{\gamma}(a, \omega)=\gamma(a) \mu_{\mathcal{G}}(\omega)
$$

By construction

$$
s \hat{\gamma}(a, \omega)=0
$$

with

$$
\begin{aligned}
s a & =\ell_{\omega} a \\
s \omega & =-\frac{1}{2}[\omega, \omega]
\end{aligned}
$$

and $\hat{\gamma}$ is ambiguous up to a coboundary:

$$
\hat{\gamma} \rightarrow \hat{\gamma}+s \chi
$$

which is a consequence of the fact that two top classes of $\mathcal{G}$ with compact support which integrate to 1 differ by a coboundary. These constructions can be taken as a basis for the geometrical origin of the Slavnov symmetry. When $\gamma$ is constructed by patching up local sections, as in eq. (3.28), one has the well known formulae

$$
\begin{aligned}
\hat{\gamma}_{i}(a, \omega) & =\delta\left(g_{i}\right) \wedge s g_{i} \\
& =\int \mathcal{D} b \mathcal{D} \bar{\omega} e^{i<b, g_{i}>-<\bar{\omega}, s g_{i}>} .
\end{aligned}
$$


Extending the operation $s$ to the integration variables

$$
\begin{aligned}
s \bar{\omega} & =i b \\
s b & =0 .
\end{aligned}
$$

This can be rewritten

$$
\hat{\gamma}_{i}(a, \omega)=\int \mathcal{D} b \mathcal{D} \bar{\omega} e^{s\left(<\bar{\omega}, g_{i}>\right)} .
$$

These formulae are valid as long as the manifolds $g_{i}=0$ stay transverse to the fibers. The Faddeev Popov operator $m_{i}$ defined by

$$
s g_{i}=m_{i} \omega
$$

is then invertible. The $\delta$ current can be smoothed out by adding a term $\langle i \bar{\omega}, \varphi(b)>$ under the $s$ operation where $\varphi$ is such that $\langle b, \varphi(b)\rangle$ is positive at infinity. This suggests a class of gauge fixing forms

$$
\hat{\gamma}(a, \omega)=\int \mathcal{D} b \mathcal{D} \bar{\omega} e^{s(<\bar{\omega}, g(a, \dot{a})>+i<\bar{\omega}, \varphi(b, \dot{a})>)}
$$

where $g(a, \dot{a})$ and $\varphi(b, \dot{a})$ involve an additional orbit space dependence such that $m(a, \dot{a})$ remains invertible and $\gamma(a, \omega)$ has compact support or fast decrease. The inclusion of such dependences is liable to spoil renormalizability or locality or both.

The situation with topological theories is slightly more involved and goes through several steps.

Orbit space is restricted to the finite dimensional manifold of gauge equivalence classes of solutions of a $\mathcal{G}$ invariant system of equations $\varepsilon$ e.g. $F^{-} \equiv F-* F=0$. This restriction is obtained via the insertion of the corresponding Mathaï Quillen form $\Theta_{\varepsilon}$. One now considers an observable of degree the dimensionality $d$ of this submanifold e.g.

$$
\mathcal{O}_{d}=\prod_{\Sigma g_{i}=d} \mathcal{O}^{\mathrm{g}_{i}}
$$

Choosing a connection $\tilde{\omega}$ with curvature $\tilde{\Omega}$ transforms $\Theta_{\varepsilon}$ into $\tilde{\Theta}_{\varepsilon}, \mathcal{O}_{d}$ into $\tilde{\mathcal{O}}_{d}$. In particular $\psi=\delta a+D_{a} \omega$ is transformed into $\tilde{\psi}=\delta a+D_{a} \tilde{\omega}$. $\tilde{\mathcal{O}}_{d} \tilde{\Theta}_{\varepsilon}$ defines a basic form which can be integrated over $\mathcal{A} / \mathcal{G}$ after choosing local sections and a partition of unity:

$$
\begin{aligned}
<\mathcal{O}_{\mid \varepsilon}>= & \int_{\mathcal{A} / \mathcal{G}} \sum_{i} \theta_{i}(\dot{a}) \sigma_{i}^{*}\left(\tilde{\mathcal{O}}_{d} \tilde{\Theta}_{\varepsilon}\right) \\
= & \int \sum_{i} \theta_{i}(\dot{a}) \mathcal{D} \omega \mathcal{D} \Omega\left(\mathcal{O}_{d} \Theta_{\varepsilon}\right)(a, \tilde{\Omega}, \tilde{\psi}) \\
& \delta(\omega-\tilde{\omega}) \delta(\Omega-\tilde{\Omega}) \delta\left(g_{i}\right) \wedge \frac{\delta g_{i}}{\delta a} D_{a} \tilde{\omega}
\end{aligned}
$$

where $\tilde{\psi}=\delta a+D_{a} \tilde{\omega}$ and $\tilde{\Omega}$ is quadratic in $\tilde{\psi}$. In the last factor one can write indifferently $\frac{\delta g_{i}}{\delta a} \delta a=\frac{\delta g_{i}}{\delta a}\left(\tilde{\psi}-\mathcal{D}_{a} \tilde{\omega}\right)$ or $\left.\frac{\delta g_{i}}{\delta a} D_{a} \tilde{\omega}\right)=m_{i} \tilde{\omega}$ because $\left(\mathcal{O}_{d} \Theta_{\epsilon}\right)$ has maximum degree in $\tilde{\psi}$. Using 
the $\delta(\omega-\tilde{\omega})$ factor we can replace everywhere $\tilde{\psi}$ by $\psi=\delta a-D_{a} \omega$ and, in the last factor $\tilde{\omega}$ by $\omega$. Expressing also $\delta(\omega-\tilde{\omega})$ in terms of $\psi=\delta a-D_{a} \omega$, we can rewrite

$$
\begin{aligned}
<\mathcal{O}_{\mid \varepsilon}>= & \int \sum_{i} \theta_{i}(\dot{a}) \mathcal{D} \omega \mathcal{D} \Omega\left(\mathcal{O}_{d} \Theta_{\varepsilon}\right)(a, \tilde{\Omega}(\psi), \psi) \\
& \delta((\omega-\tilde{\omega}(\psi)) \delta(\omega-\tilde{\omega}(\psi)) \\
& \mathcal{D} \psi \delta\left(\psi-\left(\delta a+D_{a} \omega\right)\right) \delta\left(g_{i}\right) \wedge m_{i} \omega
\end{aligned}
$$

Using now the fact that $\left.\left(\mathcal{O}_{d} \Theta_{\varepsilon}\right)(a, \tilde{\Omega}(\psi), \psi) \delta(\omega-\tilde{\omega})(\psi)\right)$ is of maximum degree in $\psi$ and also that $\wedge m_{i} \omega$ is of maximum degree in $\omega \delta\left(\psi-\left(\delta a+D_{a} \omega\right)\right)$ can be replaced by $\delta(\delta a)=\mathcal{D} a$. We end up with

$$
\begin{gathered}
<\mathcal{O}_{\mid \varepsilon}>\int \sum_{i} \theta_{i}(\dot{a}) \mathcal{D} \omega \mathcal{D} \Omega \mathcal{D} \psi \mathcal{D} a\left(\mathcal{O}_{d} \Theta_{\varepsilon}\right)(a, \Omega, \psi) \\
\delta((\omega-\tilde{\omega})(\psi) \delta(\Omega-\tilde{\Omega}(\psi))) \delta\left(g_{i}\right) \wedge m_{i} \omega
\end{gathered}
$$

As shown before, $\mathcal{O}_{\varepsilon} \delta((\omega-\tilde{\omega})(\psi)) \delta(\Omega-\tilde{\Omega}(\psi))$ can be expressed in terms of Fourier transforms involving pairs of fermionic and bosonic integration variables. In the present example, with

$$
\tilde{\omega}=-\frac{1}{D_{a}^{*} D_{a}} D_{a}^{*} \delta a
$$

(which excludes reducible connections),

$$
\begin{aligned}
& \Theta_{\varepsilon} \delta((\omega-\tilde{\omega})(\psi)) \delta(\Omega-\tilde{\Omega}(\psi))=\int \mathcal{D} \bar{\omega}^{-} \mathcal{D} b^{-} \mathcal{D} \bar{\omega} \mathcal{D} \bar{\Omega} \\
& e^{s^{t o p}\left(\bar{\omega}^{-} F^{-}+\bar{\Omega} D^{*} \psi\right)} \\
& =e^{i b^{-} F^{-}+\bar{\omega}^{-}(D \psi)^{-}+\bar{\omega} D^{*} \psi+i \bar{\Omega}\left(D^{*} D \Omega+\left[\psi^{+}, \psi\right]\right)} .
\end{aligned}
$$

The expression under the exponential can be supplemented by a term consistent with power counting $\operatorname{tr}[\bar{\Omega}, \Omega] \bar{\omega}$ yielding in the action the sum of two terms : $\operatorname{tr}\left\{[\bar{\Omega}, \Omega]^{2}+[\bar{\omega}, \bar{\omega}] \Omega\right\}$.

The manipulations described above provide a bridge between the interpretation of L. Baulieu, I.M. Singer 14, 15 and that of M.F. Atiyah, L. Jeffrey [17]. They may seem fairly arbitrary and non unique. They are geared towards field theory integral representations of orbit space integrals whose ultraviolet singular behaviours have to be controlled in an algebraic manner. In that respect, the gauge fixing factor lacks an algebraic characterization which extends in a satisfactory way that of the first part: the full action has the form

$$
S\left(a, \psi, \omega, \Omega ; \bar{\omega}^{-} b^{-} \bar{\omega} \bar{\Omega}\right)=s^{t o p} \chi+S_{g f}
$$

Let us recall

$$
\begin{aligned}
s^{t o p} a & =\psi-D_{a} \omega \\
s^{t o p} \psi & =[\omega, \psi]-D_{a} \Omega \\
s^{t o p} \omega & =\Omega-\frac{1}{2}[\omega, \omega] \\
s^{t o p} \Omega & =-[\omega, \Omega]
\end{aligned}
$$




$$
\begin{aligned}
s^{t o p} \bar{\omega}^{-} & =i b^{-}+\left[\omega, \bar{\omega}^{-}\right] \\
s^{t o p} i b^{-} & =\left[\omega, i b^{-}\right]-\left[\Omega, \bar{\omega}^{-}\right] \\
s^{t o p} i \bar{\Omega} & =\bar{\omega}+[\omega, i \bar{\Omega}] \\
s^{t o p} \bar{\omega} & =-[\Omega, i \bar{\Omega}]+[\omega, \bar{\omega}] \\
I(\lambda) \omega & =\lambda \quad I(\lambda) \text { other }=0 \quad \lambda \in \text { LieG } \\
L(\lambda) & =\left[I(\lambda), s^{t o p}\right]_{+}
\end{aligned}
$$

$\chi$ is basic:

$$
I(\lambda) \chi=L(\lambda) \chi=0 ; \lambda \in \operatorname{Lie} \mathcal{G} .
$$

In order to extend this structure to $S_{g f}$, we replace the basicity of $\chi$ by an equivalent condition. Define [16]

$$
W=L(\underline{\lambda})+I(\underline{\mu})
$$

where $\underline{\lambda}$ generates $\wedge(\operatorname{Lie\mathcal {G}})^{*}$ and $\underline{\mu}$ generates $S\left(\right.$ Lie $\left.^{*}\right)$. In particular

$$
\begin{aligned}
W \underline{\lambda} & =-\frac{1}{2}[\underline{\lambda}, \underline{\lambda}]_{+} \\
W \underline{\mu} & =-[\underline{\lambda}, \underline{\mu}]_{-} .
\end{aligned}
$$

By construction, $W^{2}=0$.

If one extends $s^{\text {top }}$ to the algebra generated by $\underline{\lambda}, \underline{\mu}$ according to

$$
\begin{aligned}
& s^{t o p} \underline{\lambda}=\underline{\mu} \\
& s^{t o p} \underline{\mu}=0
\end{aligned}
$$

we have

$$
\left[s^{t o p}, w\right]=0 .
$$

From now on, we shall omit the underlining of $\underline{\lambda}$ and $\mu$ which will denote the Faddeev Popov ghosts of the graded Lie algebra generated by $L(\cdot), \bar{I}(\cdot)$. It is clear that, restricted to $\lambda, \mu$ independent elements, basicity is equivalent to $W$ invariance. Thus,

$$
W \chi=0 .
$$

We now introduce Lagrange multipliers and antighosts $(\bar{\lambda}, \ell)(\bar{\mu}, m)$ associated to $\lambda, \mu$. We extend $W$ by

$$
\begin{aligned}
W \bar{\lambda} & =\ell \\
W \ell & =0 \\
W \bar{\mu} & =m \\
W m & =0
\end{aligned}
$$

$s^{t o p}$ has to be extended in order to preserve its anticommutation with $W$ :

$$
\begin{array}{ll}
s^{t o p} \bar{\mu}=\bar{\lambda} & s^{t o p} \bar{\lambda}=0 \\
s^{t o p} m=-\ell & s^{t o p} \ell=0 .
\end{array}
$$


We now have a bigrading relative to the two differentials $s^{t o p}, W$, as follows

$$
\begin{array}{rccccccccccccc} 
& s^{\text {top }} & W & a, & \psi & \omega, & \Omega & \text { etc. } & \lambda & \mu & \bar{\lambda} & \bar{\mu} & \ell & m \\
s \text { grading } & 1 & 0 & 0 & 1 & 1 & 2 & & 0 & 1 & 0 & -1 & 0 & -1 \\
W \text { grading } & 0 & 1 & 0 & 0 & 0 & 0 & & 1 & 1 & -1 & -1 & 0 & 0
\end{array}
$$

Now, we are going to prove that any $S_{g f}$ a polynomial of positive degree in the Lagrange multipliers $\Lambda=(\bar{\lambda}, \bar{\mu}, \ell, m)$ which is both $W$ and $s^{t o p}$ invariant is of the form

$$
S_{g f}=s^{t o p} W X
$$

Using the homotopy $k_{w}$ associated with the abelian Weil algebra, cf. Eq. (2.8), we can write

$$
S_{g f}=\left(k_{W} W_{\Lambda}+W_{\Lambda} k_{W}\right) S_{g f}
$$

where $W_{\Lambda}$ is the part of $W$ acting on $\Lambda$. Writing

$$
W=W_{\Lambda}+W_{0}
$$

and using

$$
W S_{g f}=0
$$

we get

$$
\begin{aligned}
S_{g f} & =\left(-k_{W} W_{0}+W_{\Lambda} k_{W}\right) S_{g f}=\left(W_{0}+W_{\Lambda}\right) k_{W} S_{g f} \\
& =W k_{W} S_{g f}
\end{aligned}
$$

We now construct a homotopy for $s^{t o p}$, extending the homotopoy of the Weil algebra: For all pairs $x, y$ such that

$$
\begin{aligned}
s^{t o p} x & =y+\ell_{\omega} x \\
s^{t o p} y & =-\ell_{\Omega} x+\ell_{\omega} y
\end{aligned}
$$

we introduce families $x_{t}$ such that

$$
\begin{aligned}
s^{t o p} x_{t} & =y_{t}+\ell_{\omega_{t}} x_{t} \\
s^{t o p} y_{t} & =-\ell_{\Omega_{t}} x_{t}+\ell_{\omega_{t}} y_{t}
\end{aligned}
$$

and we define $k_{s}(t)$

$$
\begin{aligned}
k_{s}(t) x_{t} & =0 \\
k_{s}(t) y_{t} & =d_{t} x_{t}
\end{aligned}
$$

$k_{s}$ is then defined analogously to Eq.(2.8) as

$$
k_{s}=\int_{0}^{1} k_{s}(t) \varphi_{t} .
$$


Then using

$$
s^{t o p} S_{g f}=0 .
$$

we get

$$
S_{g f}=s^{t o p} k_{s} S_{g f}
$$

Hence

$$
S_{g f}=W k_{W} s^{t o p} k_{s} S_{g f} .
$$

It is straightforward to check that $k_{W}$ and $s^{\text {top }}$ anticommute, hence

$$
\begin{aligned}
S_{g f} & =s^{t o p} W k_{W} k_{s} S_{g f} \\
& =s^{t o p} W \chi
\end{aligned}
$$

where $\chi$ has to have bigrading $(-1,-1)$ and is thus of the form

$$
\chi=<\bar{\mu}, g(a)>+(\bar{\mu}, \ell) .
$$

This gives

$$
\begin{aligned}
S_{g f}= & s^{t o p}\left(<m, g(a)>+<\bar{\mu}, \frac{\delta g}{\delta a} D_{a} \lambda>+(m, \varphi(\ell))\right) \\
= & -<\ell, g(a)>-<m, \frac{\delta g}{\delta a}\left(\psi-D_{a} \omega\right)> \\
& +<\bar{\lambda}, \frac{\delta g}{\delta a} D_{a} \lambda>+<\bar{\mu}, \frac{\delta g}{\delta a} D_{a} \mu>+ \\
& <\bar{\mu}, \frac{\delta m}{\delta \underline{a}} \cdot \psi-\underline{D_{a}} w \cdot \lambda>\cdot(\ell, \varphi(l))
\end{aligned}
$$

There remains to prove that locally over orbit space this provides a gauge fixing. So, we have to look at

$$
\int \mathcal{D} \lambda \mathcal{D} \mu \mathcal{D} \bar{\lambda} \mathcal{D} \bar{\mu} \mathcal{D} \ell \mathcal{D} m e^{S_{g f}} .
$$

First, against an integrand of top degree in $\psi$ the $\psi$ dependence of $S_{g f}$ can be forgotten. Secondly the $m$ integration insures that the second term yields $\wedge m \omega$, of maximal degree in $\omega$. The term before last can thus be forgotten, and the $\bar{\lambda} \lambda \bar{\mu} \mu$ integrations yields twice det $m$ with opposite powers. As remarked earlier one could generalize this to

$$
\chi=\bar{\mu} g(a, \dot{a})+\bar{\mu} \varphi(\ell, \dot{a}) .
$$

So, we have constructed a complicated integral representation of the standard gauge fixing form whose advantage is to allow constraining the ultraviolet ambiguities within a tight algebraic set up.

This concludes the third exercise. 


\section{CONCLUSION}

Equivariant cohomology provides an algebraic equipment quite well adapted to semi-classical formulations of gauge theories. Some integral representations associated with those have been collected in these notes, with emphasis on their algebraic structures and some of the freedom that is allowed. Similar algebraic techniques have been used recently to establish other integral representations [21] and concrete formulae [22]. Gauge fixing appears as a necessity when equivariance with respect to non compact groups comes into play, each time one is writing an integral representation over field space as opposed to orbit space. It is in general this way ultraviolet problems have been so far mastered thanks to locality. It is suggested that more general gauge fixing procedures than the conventional ones have to be used in order to respect the geometry. The question remains open whether, at the expense of introducing extra local fields, some can be found which respect both locality and renormalizability. 


\section{ACKNOWLEDGEMENTS}

These notes have benefited from numerous discussions with M. Bauer, C. Becchi, G. Girardi, C. Imbimbo, F. Thuillier, J. Zinn-Justin, R. Zucchini to whom the author expresses all his gratitude.

\section{References}

[1] Cartan H., Colloque de Topologie (Espaces fibrés), Brussels (1950) CBRM 15-56.

[2] Atiyah M.F., Bott R., 1984, Topology 23, 1.

[3] Mathaï V., Quillen D., 1986, Topology 25, 85.

[4] Kalkman J., 1993, Com. Math. Phys. 153, 447

[5] Duflo M., Kumar S., Vergne M., 1993 Asterisque 215.

[6] Witten E., 1988, CMP 117, 353

Witten E., 1991, Int. Journ. of Mod. Phys. A6, 2115.

[7] Braam P., April 1988, CERN Seminar.

[8] Faddeev L.D., Popov V.N., 1967, Phys. Lett. B25, 29.

[9] Cordes S., Moore G., Ramgoolam S., Les Houches Lectures 1994, HEPTH 941 1210, YCTP P11-94.

[10] Zumino B., Les Houches lectures 1984: in Relativity, groups and topology II; De Witt B.S., Stora R. eds, Amsterdam, North Holland 1984.

[11] Manes J., Stora R., Zumino B., 1985, CMP 102, 157.

[12] Berline N., Getzler E., Vergne M., "Heat Kernels and Dirac operators", Grundlehren des Mathematischen Wissenschaft 298, Springer Verlag Berlin Heidelberg 1992.

[13] Stora R., Thuillier F., Wallet J.C., Lectures at the 1st Caribbean Spring School of Mathematics and Theoretical Physics, Saint-François, Guadeloupe, May 30-June 15, 1994.

[14] Baulieu L., Singer I.M., Infinite dimensional geometry, non commutative geometry, operator algebras, fundamental interactions, 1988, NP B15 (Proc. Suppl.), 12.

[15] Baulieu L., Singer I.M., 1991, CMP 135253.

[16] Ouvry S., Stora R., van Baal P., 1989, Phys. Lett. B220, 159.

[17] Atiyah M.F., Jeffrey L., 1990, JGP 7, 119. 
[18] Zinn Justin J., Quantum field theory and critical phenomena, Clarendon Press, Oxford 1989, p. 485.

[19] Ruelle D., Sullivan D., 1975, Topology 14, 319

Connes A., Non Commutative Geometry, Academic Press New York (1973).

[20] Greub V., Halpern S., Vanstone R., Connections, curvature and cohomology, Vol. III Acad. Press New York (1973).

[21] Wu S., 1993, JGP 10 nj4, 381;

Becchi C., Collina R., Imbimbo C., CERN-TH-7302/94, GEF-TH-6-1994 to appear in "Symmetry and Simplicity in Theoretical Physics", Symposium for the 65th birthday of Sergio Fubini, Torino 1994, World Scientific, Singapore 1994;

Imbimbo C., Nucl. Phys. B41 (Proc. Suppl.) 302, 1995;

Becchi C., Imbimbo C., CERN-TH-95-242 GEF TH 95-8, in BRS Symmetry, Abe M., Nakanishi N., Ojima I. Ed. Universal Academy Press, Tokyo, Japan 1996.

[22] Bauer M., Thuillier F., Representatives of the Thom class of a vector bundle, in preparation. 